\title{
Defending Against Barrier Intrusions with Mobile Sensors
}

\author{
Guanqun Yang, Wei Zhou, Daji Qiao \\ Iowa State University, Ames, IA 50011, USA \\ \{gqyang,zhouwei,daji\}@iastate.edu
}

\begin{abstract}
Deploying wireless sensor networks has been recognized as one of the effective and cost-efficient ways to defend remote barrier regions such as international territorial borders. It has also been demonstrated recently that, by introducing sensor mobility into the wireless sensor network, the network coverage performance can be greatly improved. In this paper, we employ a game theoretic approach to study the problem of defending against barrier intrusions with mobile sensors, and demonstrate the equilibriums between barrier intrusions and sensor movement strategies in both non-obstructed and obstructed barriers. In particular, we derive the optimal sensor movement strategy to defend non-obstructed barriers, and propose a Dijkstra-like algorithm to generate a sensor movement strategy that performs well in defending obstructed barriers. We verify the correctness of our theoretic analysis by conducting in-depth simulations under various barrier intrusion and sensor movement scenarios.
\end{abstract}

\section{Introduction}

\subsection{Motivation and Contributions}

A major category of applications for sensor networks is border surveillance and intrusion detection, which has recently attracted substantial research interests. The common goal of these applications is to detect intruders as they cross the border or as they penetrate into a protected area. In the meantime, sensors mounted on mobile platforms (a.k.a. mobile sensors) have received increasing attention, because mobile sensor networks could be extremely valuable in certain working environments where traditional sensor deployment schemes can not be employed, for example, remote territorial borders or disaster areas.

In this paper, we investigate the problem of defending against barrier intrusions with mobile sensor networks. A barrier intrusion is generally referred to as the motion of an intruder starting from any point on the entrance side of the barrier to reach any point on the destination side. We define the intrusion coverage degree as the expected number of distinct sensors that each detects the intruder before it crosses the barrier, and take a game theoretic approach to study the best worst-case performance of a mobile sensor network in terms of the intrusion coverage degree. Note that the coverage performance of a mobile sensor network depends on the initial network configuration as well as the movement of mobile sensors. Specifically, the main contributions of this work are:

- For barriers without obstacles, we demonstrate that there exists an equilibrium between barrier intrusion and sensor movement strategy, and derive the corresponding sensor movement strategy that maximizes the minimum intrusion coverage degree among all possible intrusions to the barrier;

- For barriers with obstacles, we first identify the unique $o b$ stacle eclipse effect that can only be observed in obstructed barriers, and then propose a Dijkstra-like algorithm to generate a sensor movement strategy that reaches an intrusion/defense equilibrium under a few simplifying assumptions. Simulation results show that the devised sensor movement strategy performs well in defending obstructed barriers under practical considerations.

\subsection{Related Work}

The coverage problem in static wireless sensor networks has been studied intensively [6,16,18-20,24]. In [18], the authors studied three fundamental coverage metrics: area coverage, node coverage, and detectability. The authors of [16,24] investigated asymptotic 1 -coverage and $k$-coverage respectively in mostlysleeping wireless sensor networks. However, none of the above work studied the sensor network coverage problem when targets are mobile.

In $[6,19,20]$, the authors studied the sensor network coverage problems with regard to mobile targets and provided solutions to finding minimum-exposure paths. Intrusion detection problems in static sensor network have been addressed in $[5,7,10,15]$. Cao et al [5] calculated the detection probability and average detection delay of the intrusion. In [7], Dousse et al used the supercritical percolation model to analyze the detection delay for a straight-moving target. In [10], metrics for quality of surveillance were proposed for the network operations in both surveillance and tracking states. In [15], the authors introduced the concept of $k$-barrier coverage and derived both sufficient and necessary conditions for asymptotic $k$-barrier coverage in a belt region. In [9], the authors investigated the problem of optimizing the locations of additional static sensors to improve coverage of the maximum breach path to a protected object.

Researchers have recently started to study the coverage of mobile sensor networks [2,12,22]. However, in most of the proposed algorithms, sensor mobility was exploited essentially to obtain a new static network configuration that improves coverage after the sensors are re-positioned to their desired locations. In [17], the authors studied the coverage of a mobile sensor network from a different perspective by exploring the dynamic aspects of network coverage that depends on the movement of sensors. They proved that introduction of sensor mobility into a sensor network 
can greatly improve the network coverage performance, and derived the optimal sensor movement strategy against a free-moving target. In [3], the authors studies the problem of using mobile sensors to catch stochastic events. Specifically, they devised optimal algorithms to plan sensor movement when events occurred along a known line or closed curve. For the 2-D case where events could occur at arbitrary places on a plane, heuristic algorithms were provided. The above two studies are different from the focus of this paper, which is to investigate optimal sensor movement strategies to defend against barrier intrusions, where the ultimate goal of an intruder is to cross the barrier (in contrast to free movement) and it may follow any path in the barrier to achieve this goal.

\subsection{Organization}

The rest of this paper is organized as follows. Section 2 describes the system models of barrier intrusions and mobile sensors. Section 3 gives the problem statement and discusses two important preliminaries. Section 4 presents the details of designing optimal sensor movement strategies for both non-obstructed and obstructed barriers. Section 5 confirms our theoretic analysis with simulation results, and finally, Section 6 concludes the paper.

\section{System Models}

\subsection{Barrier Intrusions}

We model the barrier as a long belt region with two parallel sides: entrance side and destination side. A barrier may or may not be obstructed. An intrusion is defined as the motion of an intruder starting from an entry point on the entrance side to reach any point on the destination side.

Let $\phi_{t}$ and $\varphi_{t}$ denote the entry point and exit point of an intrusion, respectively. We divide the intrusion $\phi_{t} \rightsquigarrow \varphi_{t}$ into $n$ small intrusion segments, as shown in Fig. 1, and denote the $i$-th intrusion segment as $x_{i} \rightarrow x_{i+1}$, where $i=0, \ldots, n-1$. Hence, $x_{0}$ is the entry point $\phi_{t}$, and $x_{n}$ is the exit point $\varphi_{t}$. Furthermore, we assume that

- $x_{i} \rightarrow x_{i+1}$ can be approximated as a line segment with direction $\theta_{t, i}$ and length $d_{x_{i} \rightarrow x_{i+1}}$;

- The intruder moves at a constant speed $v_{t, i}$ along $x_{i} \rightarrow x_{i+1}$ in the direction of $\theta_{t, i}$.

As the number of segments $(n)$ goes to infinity, the series of segments $\phi_{t} \rightarrow x_{1} \rightarrow x_{2} \rightarrow \cdots \rightarrow \varphi_{t}$ approximates closely the intrusion $\phi_{t} \rightsquigarrow \varphi_{t}$, and hence we can uniquely identify an intrusion with its starting point $\left(\phi_{t}\right)$, exit point $\left(\varphi_{t}\right)$, point vector $\left\{x_{1}, \cdots, x_{n-1}\right\}$ and speed vector $\bar{v}_{t}=\left\{v_{t, 0}, \cdots, v_{t, n-1}\right\}$.

There are two main factors that affect the coverage degree of a barrier intrusion: (i) the length of the intruding path, and (ii) the proximity of the intruding path to the obstacles (if obstacles are present in the barrier). In general, a barrier intrusion with a shorter intruding path and/or closer to obstacles may yield a lower intrusion coverage degree. Hence, if an intruder is fully aware of

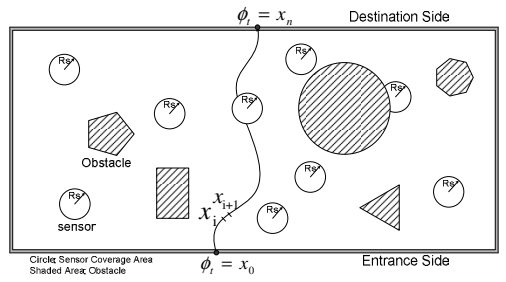

Figure 1. The intruding path is divided into $n$ segments from the entrance side to the destination side.

the barrier environment, it may plan its intrusion carefully around the obstacles to minimize the chance of being detected. Clearly, the major intrusion threat to a sensor-covered barrier comes from such smart intruders. In Section 3.2, we will discuss how the $o b$ stacle eclipses created by obstacles help smart intruders conceal themselves from being detected by sensors.

\subsection{Sensing and Coverage Models}

In this paper, for simplicity, we assume an ideal disc sensing model to demonstrate how a well-designed sensor movement strategy may help defend against barrier intrusions. Specifically, we assume that each sensor has a sensing radius of $R_{s}$, and any object within a disc of radius $R_{s}$ centered at a sensor can be detected by the sensor. The analysis on obstacle eclipses in Section 3.2 and the proposed mechanisms in Section 4 to devise optimal sensor movement strategies can be extended to more realistic sensing models such as the general sensing model described in [20].

Based on the disc sensing model, we define the intrusion coverage degree to be the expected number of distinct sensors that are able to detect the intruder as it moves along the intruding path. This is different from the conventional coverage degree defined for point coverage $[16,26]$ or barrier coverage [15] in static sensor networks.

\subsection{Sensor Deployment and Configuration}

We consider a wireless sensor network consisting of a large number of mobile sensors in a barrier. Sensors are aware of the barrier environment, for example, the locations, sizes and shapes of the obstacles in the case of an obstructed barrier.

Due to lack of control for deterministic sensor deployment [28], we assume that all the sensors are deployed according to a Poisson point process, i.e., sensor locations are modeled by a two-dimensional Poisson point process $[11,16]$. Let $\lambda$ denote the density of such Poisson point process, then the number of sensors deployed in a region $A$, denoted as $N(A)$, conforms to the following Poisson distribution:

$$
P(N(A)=k)=\frac{e^{-\lambda|| A||}(\lambda\|A\|)^{k}}{k !}
$$

where $\|A\|$ represents the area of $A$. Moreover, we assume that each deployed sensor is able to measure $[4,8,14,25]$ or esti- 
mate $[13,21,23]$ its geographical location, and then plans its movement accordingly.

\subsection{Sensor Mobility Model}

As pointed out in [17], by introducing sensor mobility into a wireless sensor network, more areas can be covered and hence the detection time of an object (static or mobile) may be shortened. In general, the movement of a sensor can be characterized by its speed and direction. We assume that each sensor moves at the fixed speed (preferably at the maximum allowed speed, in order to shorten the detection time [17]), and we assume that sensors move independently of each other. Therefore, the movement of a sensor can be characterized by a probability density function $f_{\Theta_{s}}\left(\theta_{s}\right)$ of its moving direction $\theta_{s}$. Moveover, we enforce an important zero-expected-displacement constraint on the sensor movement:

$$
\int_{0}^{2 \pi} f_{\Theta_{s}}\left(\theta_{s}\right) \cos \theta_{s} d \theta_{s}=\int_{0}^{2 \pi} f_{\Theta_{s}}\left(\theta_{s}\right) \sin \theta_{s} d \theta_{s}=0,
$$

which is a necessary condition in order to keep all deployed sensors within the boundary of the barrier over time.

\section{Problem Statement and Prelimaries}

\subsection{Problem Statement}

In this paper, we take a game theoretic approach to study the best worst-case scenarios in defending against intrusions in both non-obstructed and obstructed barriers with mobile sensors. Specifically, our goals are, for a given barrier of interest, to analyze the best intrusion to cross the barrier with minimum intrusion coverage degree, and to design a sensor movement strategy to maximize the minimum intrusion coverage degree among all possible intrusions to the barrier.

Next, before delving into the details on how to design sensors' movement strategies, we first discuss the unique obstacle eclipse effect in obstructed barriers and calculation details of the intrusion coverage degree.

\subsection{Definition of "Obstacle Eclipse"}

Obstacle eclipse is a unique phenomenon that can only be observed when there are obstacles in the sensing field. To study the sensor network coverage in obstructed barriers, it is critical to have a good understanding of the formulation and characteristics of obstacle eclipses. Formally, the obstacle eclipse of an object is defined as follows.

Definition 1 The obstacle eclipse of an object is such a region that the distance between the object and any point inside the region is less than the sensing radius, but the line of sight between them is obstructed by the obstacles.

In other words, any sensor deployed inside the obstacle eclipse (if possible) is not able to sense the object; however, if the obstacles are removed from the sensing field, this sensor shall be able to sense the object because the object is within its sensing radius. Obstacles themselves are considered part of the obstacle eclipse. The obstacle eclipse varies with the position of the object and the locations/sizes/shapes of the obstacles, as well as the sensing radius. As shown in Fig. 2(a), sensor B is inside the object's obstacle eclipse (shown as the shaded region) and hence cannot sense the object even though the distance between them is less than the sensing radius. In contrast, sensor A is outside the obstacle eclipse and can sense the object. The obstacle eclipse effectively reduces the number of sensors that can sense the object. Hence, it could be beneficial for an intruder to plan its intrusion around the obstacles because such an intrusion may yield a lower coverage degree due to the obstacle eclipse effect.

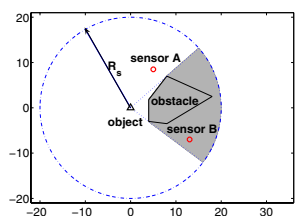

(a)

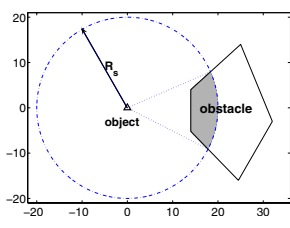

(b)
Figure 2. Illustration of obstacle eclipses. The object is shown as the small triangle point at $(0,0)$, and obstacle eclipses are shown as the shaded regions.

The above definition of the obstacle eclipse is based on the assumption of Boolean disc sensing model and line-of-sight sensing operations. With more realistic sensing models, the obstacle eclipse can be defined similarly, which is not discussed in this paper due to space limitation.

\subsection{Calculation of Intrusion Coverage Degree}

Recall that we divide an intrusion $\phi_{t} \rightsquigarrow \varphi_{t}$ into $n$ small intrusion segments, and as $n$ goes to infinity, the series of segments $\phi_{t} \rightarrow x_{1} \rightarrow x_{2} \rightarrow \cdots \rightarrow \varphi_{t}$ approximates closely the intrusion $\phi_{t} \rightsquigarrow \varphi_{t}$. Furthermore, as $n$ goes to infinity, it is reasonable to assume that the obstacle eclipse of the $i$-th segment, denoted as $\mathcal{E}\left(x_{i} \rightarrow x_{i+1}\right)$, can be approximated as the obstacle eclipse of any point on the segment; for example, $\mathcal{E}\left(x_{i} \rightarrow x_{i+1}\right)=\mathcal{E}\left(x_{i}\right)$.

In [17], the authors analyzed the expected detection time $T$ of a mobile object in a sensor network within which (i) there are no obstacles; (ii) the locations of the sensors follow a Poisson point process; and (iii) sensors and object are moving with constant speeds of $v_{s}$ and $v_{t}$, respectively. The authors concluded that $T$ follows an exponential distribution with parameter $2 \lambda R_{s} \bar{v}_{e}$, so that:

$$
P(T>t)=e^{-2 \lambda R_{s} \bar{v}_{e} t},
$$

where $\lambda$ is the density of the Poisson point process, $R_{s}$ is the sensing radius, and $\bar{v}_{e}$ is a function of $v_{s}$ and $v_{t}$ which represents the relative speed of nearby sensors to the object. In other words, for any given time interval $t$, the number of sensors that are able to detect the object during $t$ follows a Poisson distribution with density $2 \lambda R_{s} \bar{v}_{e} t$. Hence, the expected intrusion coverage degree of the $i$-th segment of an intrusion to a non-obstructed barrier is 


$$
\mathcal{C}_{x_{i} \rightarrow x_{i+1}}=2 \lambda R_{s} \bar{v}_{e, i} \frac{d_{x_{i} \rightarrow x_{i+1}}}{v_{t, i}} .
$$

On the other hand, in an obstructed barrier, due to the obstacle eclipse effect, the expected intrusion coverage degree of the $i$-th segment of an intrusion can be approximated as

$$
\mathcal{C}_{x_{i} \rightarrow x_{i+1}} \approx 2 \lambda R_{s} \bar{v}_{e, i} \frac{d_{x_{i} \rightarrow x_{i+1}}}{v_{t, i}} \cdot \frac{\pi R_{s}^{2}-\left\|\mathcal{E}\left(x_{i}\right)\right\|}{\pi R_{s}^{2}} .
$$

The coverage degree of an intrusion $\phi_{t} \rightsquigarrow \varphi_{t}$ is simply the sum of expected coverage degrees of all its segments:

$$
\mathcal{C}_{\phi_{t} \rightsquigarrow \varphi_{t}}=\sum_{i=0}^{n-1} \mathcal{C}_{x_{i} \rightarrow x_{i+1}} .
$$

It is interesting to see that a longer intruding path does not necessarily yield a higher intrusion coverage degree, especially when there are obstacles in the barrier. This is because, in addition to the path length (reflected by $d_{x_{i} \rightarrow x_{i+1}}$ ), the intrusion coverage degree is also affected by the relative speed of nearby sensors to the intrusion (reflected by $\bar{v}_{e, i}$ ), and the obstacle eclipses along the path (reflected by $\left.\left\|\mathcal{E}\left(x_{i}\right)\right\|\right)$.

\section{Sensor Movement Strategies}

We investigate the sensor movement strategies in both nonobstructed and obstructed barriers. In Section 4.1, we study the barriers without obstacles and derive the corresponding optimal sensor movement strategy. In Section 4.2, we study the barriers with obstacles and present a Dijkstra-like algorithm to generate a sensor movement strategy that performs well in defending obstructed barriers.

\subsection{Non-Obstructed Barriers}

Lemma 1 Given a sensor movement strategy, an intrusion at the maximum intruding speed $\left(\bar{v}_{t}^{\max }\right)$ always yields a lower intrusion coverage degree, in both non-obstructed and obstructed barriers, than any other intrusion along the same path but with lower speeds, i.e.,

$$
\forall f_{\Theta_{s}}, \phi_{t}, \bar{\theta}_{t}, \bar{v}_{t}, \mathcal{C}\left(f_{\Theta_{s}}, \phi_{t}, \bar{\theta}_{t}, \bar{v}_{t}^{\max }\right) \leqslant \mathcal{C}\left(f_{\Theta_{s}}, \phi_{t}, \bar{\theta}_{t}, \bar{v}_{t}\right) .
$$

where $\bar{v}_{t}^{\max }=\left(v_{t}^{\max }, \cdots, v_{t}^{\max }\right)$.

Proof of Lemma 1 is omitted due to space limitation.

Theorem 1 Consider an intrusion with a perpendicular intruding path $\left(\bar{\theta}_{t}^{\perp}\right)$ and the maximum intruding speed $\left(\bar{v}_{t}^{\max }\right)$ in a nonobstructed barrier. An optimal sensor movement strategy to maximize the coverage degree of such an intrusion is that each sensor moves in parallel to the barrier sides according to

$$
f_{\Theta_{s}}^{=}\left(\theta_{s}\right)= \begin{cases}1 / 2, & \theta_{s}=0 \text { or } \pi, \\ 0, & \text { else. }\end{cases}
$$

Proof: Recall that the direction of the $i$-th intrusion segment is $\theta_{t, i}$. Hence, a perpendicular intruding path implies that $\left(\theta_{t, 0}, \cdots, \theta_{t, n-1}\right)=\left(\frac{\pi}{2}, \cdots, \frac{\pi}{2}\right) \equiv \bar{\theta}_{t}^{\perp}$. The problem of finding an optimal sensor movement strategy to maximize the coverage degree of such an intrusion can be depicted as the following

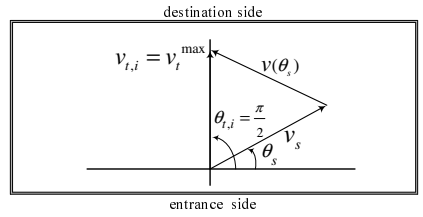

Figure 3. Illustration of the relative sensors-to-intruder speed.

optimization problem subject to the zero-expected-displacement constraint:

$$
\begin{aligned}
& \text { Given: }\left(\theta_{t, 0}, \ldots, \theta_{t, n-1}\right)=\left(\frac{\pi}{2}, \cdots, \frac{\pi}{2}\right) \equiv \bar{\theta}_{t}^{\perp} \text { and } \\
& \left(v_{t, 0}, \ldots, v_{t, n-1}\right)=\left(v_{t}^{\max }, \cdots, v_{t}^{\max }\right) \equiv \bar{v}_{t}^{\max } \\
& \max _{f_{\Theta}} \mathcal{C}\left(f_{\Theta_{s}}, \phi_{t}, \bar{\theta}_{t}^{\perp}, \bar{v}_{t}^{\text {max }}\right) \equiv \max _{f_{\Theta}} \sum_{i=0}^{n-1}\left(2 \lambda R_{s} \bar{v}_{e, i} \frac{d_{x_{i} \rightarrow x_{i+1}}}{v_{t, i}}\right) \\
& \text { subject to } E_{f_{\Theta_{s}}}\left[\cos \left(\theta_{s}\right)\right]=E_{f_{\Theta_{s}}}\left[\sin \left(\theta_{s}\right)\right]=0 .
\end{aligned}
$$

Note that $R_{s}$ is the sensing radius, $d_{x_{i} \rightarrow x_{i+1}}=\frac{d_{\phi_{t} \leadsto \varphi_{t}}}{n}$ regardless of the entrance point $\phi_{t}$, and $v_{t, i}=v_{t}^{\max }$. It is easy to verify that, for any $i$ from 0 to $n-1$,

$$
\max _{f_{\Theta_{s}}} \bar{v}_{e, i} \Longrightarrow \max _{f_{\Theta_{s}}} \sum_{i=0}^{n-1} \bar{v}_{e, i} \Longrightarrow \max _{f_{\Theta_{s}}} \mathcal{C}\left(f_{\Theta_{s}}, \phi_{t}, \bar{\theta}_{t}^{\perp}, \bar{v}_{t}^{\max }\right) .
$$

Hence, we know that a solution to the following optimization problem must be a solution to (9):

$$
\begin{aligned}
& \text { Given } \theta_{t, i}=\frac{\pi}{2}, v_{t, i}=v_{t}^{\max }, \forall i=0, \cdots, n-1, \\
& \max _{f_{\Theta_{s}}} \mathcal{V}_{i} \equiv \max _{f_{\Theta_{s}}} \bar{v}_{e, i} \\
& \text { subject to } E_{f_{\Theta_{s}}}\left[\cos \left(\theta_{s}\right)\right]=E_{f_{\Theta_{s}}}\left[\sin \left(\theta_{s}\right)\right]=0 .
\end{aligned}
$$

As shown in Fig. 3, the relative speed of nearby sensors to the intruder at the $i$-th intrusion segment, $\bar{v}_{e, i}$, can be calculated as:

$$
\begin{aligned}
\bar{v}_{e, i} & =\int_{0}^{2 \pi} \sqrt{v_{s}^{2}+v_{t, i}^{2}-2 v_{s} v_{t, i} \cos \left(\theta_{s}-\theta_{t, i}\right)} f_{\Theta_{s}}^{i}\left(\theta_{s}\right) d \theta_{s} \\
& =E_{f_{\Theta_{s}}^{i}}\left[\sqrt{v_{s}^{2}+\left(v_{t}^{\max }\right)^{2}-2 v_{s} v_{t}^{\max } \sin \left(\theta_{s}\right)}\right],
\end{aligned}
$$

where $f_{\Theta_{s}}^{i}\left(\theta_{s}\right)=\frac{1}{m} \sum_{k=1}^{m} f_{\Theta_{s}}^{u_{i, k}}\left(\theta_{s}\right)$ and $u_{i, 1}, \ldots, u_{i, m}$ are the nearby sensors to the $i$-th segment, i.e., the sensors that are within $R_{s}$ distance to the segment.

Consider another set of optimization problems:

$$
\begin{aligned}
& \forall i=0, \cdots, n-1, \\
& \max _{f_{\Theta}} \hat{\mathcal{V}}_{i} \equiv \max _{f_{\Theta_{s}}} E_{f_{\Theta_{s}}^{i}}\left[\sqrt{v_{s}^{2}+\left(v_{t}^{\max }\right)^{2}-2 v_{s} v_{t}^{\max } \sin \left(\theta_{s}\right)}\right] \\
& \text { subject to } E_{f_{\Theta_{s}}}\left[\sin \left(\theta_{s}\right)\right]=0 .
\end{aligned}
$$

Because (13) has fewer constraints than (11), we have $\max _{f_{\Theta_{s}}} \mathcal{V}_{i} \leqslant \max _{f_{\Theta_{s}}} \hat{\mathcal{V}}_{i}$. Now let $z=\sin \left(\theta_{s}\right)$, (13) becomes

$$
\begin{aligned}
& \forall i=0, \cdots, n-1, \\
& \max _{f_{\Theta_{s}}} \hat{\mathcal{V}}_{i} \equiv \max _{f_{\Theta_{s}}} E_{f_{\Theta_{s}}^{i}}\left[\sqrt{v_{s}^{2}+\left(v_{t}^{\max }\right)^{2}-2 v_{s} v_{t}^{\max z} z}\right] \\
& \text { subject to } E_{f_{\Theta_{s}}}[z]=0 .
\end{aligned}
$$

Since $g(z)=\sqrt{v_{s}^{2}+\left(v_{t}^{\max }\right)^{2}-2 v_{s} v_{t}^{\max } z}$ is a concave function of $z$ (i.e., $g^{\prime \prime}(z)<0$ ), by Jensen's inequality, we have

$$
E_{f_{\Theta_{s}}^{i}}[g(z)] \leqslant g\left(E_{f_{\Theta_{s}}^{i}}[z]\right)=g(0)=\sqrt{v_{s}^{2}+\left(v_{t}^{\max }\right)^{2}},
$$


where equality holds with probability 1 when $z=E_{f_{\Theta_{s}}^{i}}[z]=0$, or equivalently, when $\theta_{s} \in\{0, \pi\}$.

Since $f_{\Theta_{s}}$ given in (8) satisfies the constraints of (11) with the corresponding objective functions of $\forall i, \mathcal{V}_{i}=\bar{v}_{e, i}=$ $\sqrt{v_{s}^{2}+\left(v_{t}^{\max }\right)^{2}}$, and we know that $\forall i, \max _{f_{\Theta_{s}}} \mathcal{V}_{i} \leqslant$ $\max _{f_{\Theta s}} \hat{\mathcal{V}}_{i}=\sqrt{v_{s}^{2}+\left(v_{t}^{\max }\right)^{2}}$, hence $f_{\Theta_{s}}$ must be a solution to (11) and $\forall i, \max _{f_{\Theta_{s}}} \mathcal{V}_{i}=\sqrt{v_{s}^{2}+\left(v_{t}^{\max }\right)^{2}}$. Subsequently, $f_{\Theta_{s}}^{\overline{\theta_{s}}}$ is also a solution to the original optimization problem (9).

Corollary 1 The sensor movement strategy, $f_{\Theta_{s}}$, described in (8) maximizes the minimum intrusion coverage degree among all possible intrusions to a non-obstructed barrier.

Proof: (sketch) From Theorem 1, we have

$$
\forall \phi_{t}, \mathcal{C}\left(f_{\Theta_{s}}^{=}, \phi_{t}, \bar{\theta}_{t}^{\perp}, \bar{v}_{t}^{\max }\right)=\max _{f_{\Theta_{s}}} \mathcal{C}\left(f_{\Theta_{s}}, \phi_{t}, \bar{\theta}_{t}^{\perp}, \bar{v}_{t}^{\max }\right) .
$$

We have also proved in [27] that, when sensors move according to $f_{\Theta_{s}}$, an intrusion along a perpendicular path yields a lower intrusion coverage degree than any other intrusion, assuming the same intruding speed vector:

$$
\forall \phi_{t}, \bar{\theta}_{t}, \bar{v}_{t}, \mathcal{C}\left(f_{\Theta_{s}}^{=}, \phi_{t}, \bar{\theta}_{t}^{\perp}, \bar{v}_{t}\right) \leqslant \mathcal{C}\left(f_{\Theta_{s}}^{=}, \phi_{t}, \bar{\theta}_{t}, \bar{v}_{t}\right) .
$$

Combining (17) with Lemma 1, we have

$$
\forall \phi_{t}, \mathcal{C}\left(f_{\Theta_{s}}^{=}, \phi_{t}, \bar{\theta}_{t}^{\perp}, \bar{v}_{t}^{\max }\right)=\min _{\bar{\theta}_{t}, \bar{v}_{t}} \mathcal{C}\left(f_{\Theta_{s}}^{=}, \phi_{t}, \bar{\theta}_{t}, \bar{v}_{t}\right) .
$$

From (16) and (18), we have the following relation:

$$
\begin{aligned}
& \min _{\phi_{t}, \bar{\theta}_{t}, \bar{v}_{t}} \mathcal{C}\left(f_{\Theta_{s}}^{=}, \phi_{t}, \bar{\theta}_{t}, \bar{v}_{t}\right)=\max _{f_{\Theta_{s}}} \mathcal{C}\left(f_{\Theta_{s}}, \phi_{t}, \bar{\theta}_{t}^{\perp}, \bar{v}_{t}^{\max }\right) \\
& \geqslant \max _{f_{\Theta s}} \min _{\phi_{t}, \bar{\theta}_{t}, \bar{v}_{t}} \mathcal{C}\left(f_{\Theta_{s}}, \phi_{t}, \bar{\theta}_{t}, \bar{v}_{t}\right) \text {, }
\end{aligned}
$$

meaning that the parallel sensor movement strategy described in (8) maximizes the minimum intrusion coverage degree among all possible intrusions to a non-obstructed barrier.

Equilibrium From the above discussions, we conclude that a defense/intrusion equilibrium in a non-obstructed barrier is reached when (i) each sensor moves in parallel to the destination side of barrier according to $f_{\bar{\Theta}_{s}}$ described in (8); and (ii) the intruder crosses the barrier at the maximum intruding speed and along the path that is perpendicular to the destination side of the barrier. This is because any other intrusion yields a higher intrusion coverage degree, while any other sensor movement strategy allows the intruder to plan a better intrusion that yields a lower coverage degree. Hence, an equilibrium is reached.

\section{$4.2 \quad$ Obstructed Barriers}

Due to the obstacle eclipse effect, it is very difficult to derive the optimal sensor movement strategy to defend an obstructed barrier. So instead, we make a few simplifying assumptions and propose a Dijkstra-like algorithm to produce the best sensor movement strategy under those assumptions, and then use it as the approximation.

\subsubsection{Assumptions}

Firstly, we assume that the entire barrier is tiled with an eightconnected grid, as shown in Fig. 4(a), and an intruder can only move along the line segments between centers (denoted by $M$ ) of neighboring grid squares. As shown in Fig. 4(c), there are eight possible intersection points (called $K$ points in Fig. 4(b)) of an intrusion and the edges of a grid square. Note that $K$ points from neighboring grid squares may represent the same physical point. For example, $K_{i, j-1}(6), K_{i-1, j}(3)$, and $K_{i-1, j-1}(8)$ all represent the same point as $K_{i, j}(1)$. Secondly, we assume that all sensors deployed in the same grid square adopt the same movement strategy, and we use $f_{\Theta_{s},\langle i, j\rangle}$ to denote the movement strategy for sensors in grid square $\langle i, j\rangle$. Thirdly, we assume that the coverage degree of an intrusion segment in a grid square is only affected by the sensors deployed in that grid square.

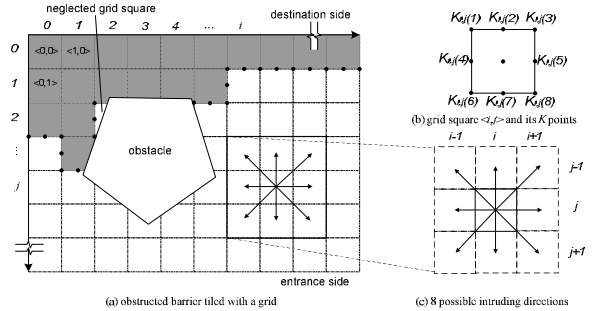

Figure 4. A snapshot of tiled obstructed barrier and structure of grid square $\langle i, j\rangle$

\subsubsection{Dijkstra-like Algorithm}

Fig. 5 shows the pseudo-code of our proposed Dijkstra-like algorithm. Set $U$ (dark region in Fig. 4(a)) consists of grid squares that have been processed and whose best sensor movement strategies have already been determined. It is initially set to empty. Set $V$ consists of grid squares that belong to $\bar{U}$ but are neighbors to $U$. Initially, it includes all the grid squares along the destination side. Set $B$ (black dots in Fig. 4(a)) consists of all the $K$ points along the boundary between $U$ and $V$. The basic idea of our algorithm is to process all grid squares recursively from the destination side to the entrance side as follows. For each grid square in $V$, the algorithm finds out the best sensor movement strategy for this grid that yields the maximin expected intrusion coverage degree among all possible intruding paths from those of its $K$ points which do not belong to $B$ to the destination side of the barrier (Lines 8-11). Then, among all grid squares in $V$, the algorithm (i) selects the grid square $\left\langle i^{*}, j^{*}\right\rangle$ whose best sensor movement strategy, denoted by $f_{\Theta_{s},\left\langle i^{*}, j^{*}\right\rangle}^{\max }$, results in the lowest maximin expected intrusion coverage degree (Line 12); (ii) plans the sensor movement in this grid square according to $f_{\Theta_{s},\left\langle i^{*}, j^{*}\right\rangle}^{\max }$ (Line 13); (iii) records the minimum expected coverage degree among all possible intruding paths from each of its $K$ points to the destination side, which is denoted by $\mathcal{C}_{J_{i^{*}, j^{*}}(\cdot) \rightsquigarrow \mid}$ (Lines 1415); and (iv) adds $\left\langle i^{*}, j^{*}\right\rangle$ to $U$, then updates $V$ and $B$ accordingly (Lines 16-18). The computation details of the intrusion coverage degree were discussed in Section 3.3, and we assume maximum 


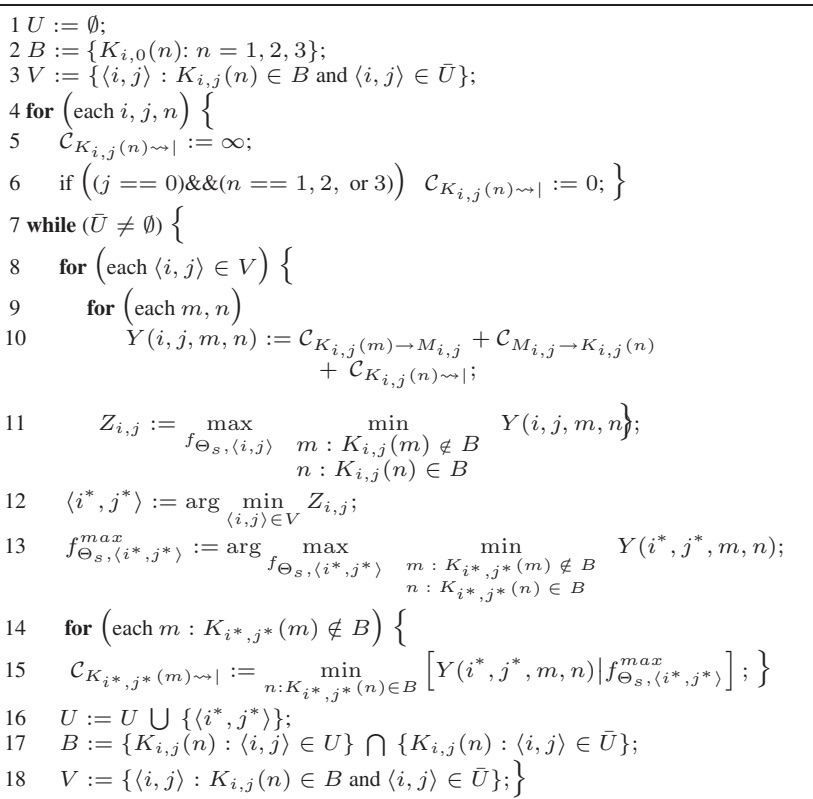

Figure 5. Pseudo-code of the proposed Dijkstra-like algorithm

intruding speed in the computation. The algorithm stops when all grid squares have been processed.

Let $\bar{f}_{\Theta_{s}}^{\max }$ denote the sensor movement strategy produced by the above Dijkstra-like algorithm, and we have the following theorem:

Theorem 2 Under the assumptions in Section 4.2.1, $\bar{f}_{\Theta_{s}}^{\max }$ maximizes the minimum intrusion coverage degree among all possible intrusions at the maximum intruding speed to an obstructed barrier.

Proof: For any given sensor movement strategy $f_{\Theta_{s}}$ and any given intruding speed vector $\bar{v}_{t}$, we use $\phi_{t}^{*}\left(f_{\Theta_{s}}, \bar{v}_{t}\right)$ to denote the entry point of the corresponding best intrusion with the minimum coverage degree. Then, for any given $f_{\Theta_{s}}$, we have

$$
\begin{aligned}
& \min _{\phi_{t}, \bar{\theta}_{t}} \mathcal{C}\left(f_{\Theta_{s}}, \phi_{t}, \bar{\theta}_{t}, \bar{v}_{t}^{\max }\right) \leqslant \min _{\bar{\theta}_{t}} \mathcal{C}\left(f_{\Theta_{s}}, \phi_{t}^{*}\left(\bar{f}_{\Theta_{s}}^{\max }, \bar{v}_{t}^{\max }\right), \bar{\theta}_{t}, \bar{v}_{t}^{\max }\right) \\
& \leqslant \min _{\bar{\theta}_{t}} \mathcal{C}\left(\bar{f}_{\Theta_{s}}^{\max }, \phi_{t}^{*}\left(\bar{f}_{\Theta_{s}}^{\max }, \bar{v}_{t}^{\max }\right), \bar{\theta}_{t}, \bar{v}_{t}^{\max }\right) \\
& =\min _{\phi_{t}, \bar{\theta}_{t}} \mathcal{C}\left(\bar{f}_{\Theta_{s}}^{\max }, \phi_{t}, \bar{\theta}_{t}, \bar{v}_{t}^{\max }\right)
\end{aligned}
$$

which means that $\bar{f}_{\Theta_{s}}^{\max }$ maximizes the minimum intrusion coverage degree among all possible intrusions at the maximum speed to an obstructed barrier. Note that the second inequality in (19) is a direct result of the proposed Dijkstra-like algorithm, as $\bar{f}_{\Theta_{s}}^{\max }$ is designed to maximize the minimum coverage degree among all possible maximum-speed intrusions starting from the same entry point.

Corollary 2 Under the assumptions in Section 4.2.1, $\bar{f}_{\Theta_{s}}^{\max } \max$ imizes the minimum intrusion coverage degree among all possible intrusions to an obstructed barrier.

Proof: $\quad$ For any given sensor movement strategy $f_{\Theta_{s}}$ and any given intruding speed vector $\bar{v}_{t}$, let $\phi_{t}^{*}\left(f_{\Theta_{s}}, \bar{v}_{t}\right)$ and
$\bar{\theta}_{t}^{*}\left(f_{\Theta_{s}}, \bar{v}_{t}\right)$ denote, respectively, the entry point and the intruding path of the corresponding best intrusion with the minimum coverage degree. Then, for any given $\bar{v}_{t}$, we have the following relation:

$$
\begin{aligned}
& \min _{\phi_{t}, \bar{\theta}_{t}} \mathcal{C}\left(\bar{f}_{\Theta s}^{\max }, \phi_{t}, \bar{\theta}_{t}, \bar{v}_{t}\right)=\mathcal{C}\left(\bar{f}_{\Theta_{s}}^{\max }, \phi_{t}^{*}\left(\bar{f}_{\Theta_{s}}^{\max }, \bar{v}_{t}\right), \bar{\theta}_{t}^{*}\left(\bar{f}_{\Theta s}^{\max }, \bar{v}_{t}\right), \bar{v}_{t}\right) \\
& \geqslant \mathcal{C}\left(\bar{f}_{\Theta s}^{\max }, \phi_{t}^{*}\left(\bar{f}_{\Theta_{s}}^{\max }, \bar{v}_{t}\right), \bar{\theta}_{t}^{*}\left(\bar{f}_{\Theta_{s}}^{\max }, \bar{v}_{t}\right), \bar{v}_{t}^{\max }\right) \\
& \geqslant \min _{\phi_{t}, \bar{\theta}_{t}} \mathcal{C}\left(\bar{f}_{\Theta_{s}}^{\max }, \phi_{t}, \bar{\theta}_{t}, \bar{v}_{t}^{\max }\right) \geqslant \max _{f_{\Theta_{s}} \min _{t}, \bar{\theta}_{t}} \mathcal{C}\left(f_{\Theta_{s}}, \phi_{t}, \bar{\theta}_{t}, \bar{v}_{t}^{\max }\right) \\
& \geqslant \max _{f_{\Theta_{s}} \phi_{t}, \bar{\theta}_{t}, \bar{v}_{t}} \mathcal{C}\left(f_{\Theta_{s}}, \phi_{t}, \bar{\theta}_{t}, \bar{v}_{t}\right) .
\end{aligned}
$$

The first and third inequalities in (20) result from Lemma 1 and Theorem 2, respectively. Hence,

$$
\min _{\phi, \bar{\theta}_{t}, \bar{v}_{t}} \mathcal{C}\left(\bar{f}_{\Theta_{s}}^{\max }, \phi_{t}, \bar{\theta}_{t}, \bar{v}_{t}\right) \geqslant \max _{f_{\Theta_{s}}} \min _{\phi_{t}, \bar{\theta}_{t}, \bar{v}_{t}} \mathcal{C}\left(f_{\Theta_{s}}, \phi_{t}, \bar{\theta}_{t}, \bar{v}_{t}\right),
$$

which means that the sensor movement strategy $\bar{f}_{\Theta_{s}}^{\max }$ maximizes the minimum intrusion coverage degree among all possible intrusions to an obstructed barrier.

Equilibrium From the above discussions, we conclude that, under the assumptions in Section 4.2.1, a defense/intrusion equilibrium in an obstructed barrier is reached when (i) sensors move according to $\bar{f}_{\Theta_{s}}^{\max }$ produced by the Dijkstra-like algorithm; and (ii) the intruder crosses the barrier at the maximum intruding speed and along the path specified by $\phi_{t}^{*}\left(\bar{f}_{\Theta_{s}}^{\max }, \bar{v}_{t}^{\max }\right)$ and $\bar{\theta}_{t}^{*}\left(\bar{f}_{\Theta_{s}}^{\max }, \bar{v}_{t}^{\max }\right)$. This is because any other intrusion yields a higher intrusion coverage degree, while any other sensor movement strategy allows the intruder to plan a better intrusion that yields a lower coverage degree. Hence, an equilibrium is reached.

\subsubsection{Practical Considerations}

In practice, since an intrusion segment will be covered by all the sensors within a circle centered at the segment and with radius of $R_{s}$, the third assumption in Section 4.2.1 does not hold. Hence, $\bar{f}_{\Theta_{s}}^{\max }$ may not be able to result in the true equilibrium. In next section, we evaluate the performance of $\bar{f}_{\Theta_{s}}^{\max }$ with different grid square sizes and simulation results show that it performs well in defending obstructed barriers under practical considerations. Moreover, we assume that mobile sensors work independently of each other. In practice, it would be beneficial by fully exploiting the cooperation among mobile sensors, although the system design may be more complicated.

In our simulation study, sensor movement is planned in rounds. Specifically, time is divided into short rounds. During the first half of each round, a sensor moves in a randomly-chosen direction $\theta_{s} \in[0,2 \pi)$ according to its movement strategy $f_{\Theta_{s}}\left(\theta_{s}\right)$; during the second half of the round, the sensor moves back towards the starting point along the direction of $\left(180^{\circ}+\theta_{s}\right)$. In the case when a sensor movement is blocked by the obstacles, it bounces back towards the starting point and, once reaching the starting point, it starts a new round. 


\section{Performance Evaluation}

\subsection{Simulation Setup}

We use the Qualnet simulator [1] to evaluate the performances of $f_{\Theta_{s}}^{=}$and $\bar{f}_{\Theta_{s}}^{\max }$ in non-obstructed and obstructed barriers, respectively. The simulated barrier is a (160 unit $\times 80$ unit) rectangle area with sensors deployed inside the barrier according to a Poisson point process with $\lambda=0.078$ per unit square.

When studying the obstructed barrier, we assume that all obstacles are in rectangular shape with various dimensions, and no sensor can be deployed inside the obstacles. Fig. 6 shows the simulated obstructed barrier. Furthermore, we assume that the sensing range is $R_{s}=2.5$ units, the sensor speed is fixed at 4 units/sec, and each round of sensor movement is 4 seconds. We evaluate and compare the coverage performance of our proposed sensor movement strategies against the random sensor movement strategy: $f_{\Theta_{s}}^{r}\left(\theta_{s}\right)=\frac{1}{2 \pi}, \forall \theta_{s} \in[0,2 \pi)$, and the static strategy where all sensors remain static. We study various types of intrusions with different entry points, speed vectors, and direction vectors. The intruding speed mav vary from 1 unit/sec to 4 units/sec.

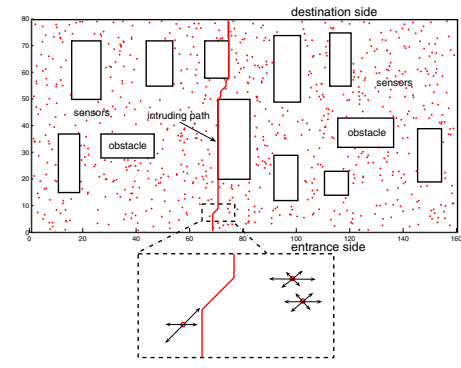

Figure 6. Simulated obstructed barrier

\subsection{Simulation Results}

\subsubsection{Impact of Sensor Movement}

We first study the effects of sensor movement on the coverage performance. For a non-obstructed barrier, we simulate 10 different sensor network deployments, and, for each deployment, we simulate 50 different entry points. The intruding speed is 1 unit/sec. For each entry point of a given sensor network deployment, the performance of a testing sensor movement strategy is characterized by the maximin intrusion coverage degree among all possible intrusions starting from that entry point. Fig. 7(a) plots the Empirical Cumulative Distribution Function (ECDF) of the normalized performance difference between each testing scheme and $f_{\Theta_{s}}$. The figure reads that $f_{\Theta_{s}}$ outperforms random sensor movement strategy and static strategy by at least $20 \%$ and $55 \%$, respectively. We perform similar simulations on the obstructed barrier (shown in Fig. 6). Since it is difficult to derive the global optimal sensor movement strategy for obstructed barriers, we plot the ECDF of the normalized performance difference between each testing scheme and a local greedy sensor movement strategy, which assumes that each sensor is aware of the intruding path, and hence can plan its movement accordingly to maximize the intrusion coverage degree. The performance of such local greedy strategy is better than that of the global optimal strategy, since the goal of the global optimal strategy is to maximin the intrusion coverage degree among all possible intrusions from any entry point. Fig. 7(b) shows the ECDF results for the obstructed barrier, and $\bar{f}_{\Theta_{s}}^{\max }$ outperforms both random and static strategies. Moreover, the performance of $\bar{f}_{\Theta_{s}}^{\max }$ varies with the grid square size, denoted as (L units $\times \mathrm{L}$ units). For this particular obstructed barrier, grid square size of $(0.5$ unit $\times 0.5$ unit $)$ seems to be a reasonable choice and will be used in other relevant simulation runs.

In general, we can see that the coverage performance is greatly improved by introducing sensor movement into the network and our proposed sensor movement strategy based on game theoretic approaches yields larger performance gain than the random strategy in both obstructed and non-obstructed barriers.

\subsubsection{Non-Obstructed Barrier}

Fig. 8 compares the coverage degrees of various straight-line intrusions in a non-obstructed barrier. The $\mathrm{x}$-axis represents the intruding angle $\theta_{t}$ and the $y$-axis represents the the normalized intrusion coverage degree over the result when $\theta_{t}=90^{\circ}$ and $v_{t}=1.0 \mathrm{unit} / \mathrm{sec}$. The intruder may move at the fixed speed of 1,2 or 4 units/sec, or vary its speed between 1 and 4 units/sec. All sensors move according to $f_{\Theta_{s}}$. Each point in the figure is averaged over 10 simulation runs. As shown in the figure, the minimum intrusion coverage degree is achieved when $\theta_{t}=90^{\circ}$ and $v_{t}=4$ units/sec. This is intuitively true since both a smaller intruding angle and a lower intruding speed result in a longer exposure time of the intruder in the barrier, and hence, a higher intrusion coverage degree. The above observations confirm our analysis in Section 4.1.

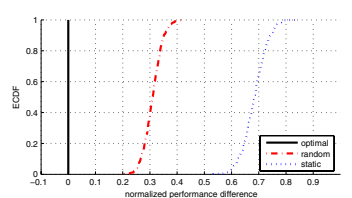

(a) non-obstructed barrier

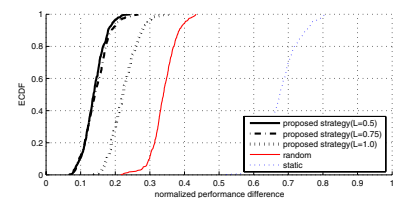

(b) obstructed barrier
Figure 7. Comparison of different sensor movement strategies.

\subsubsection{Obstructed Barrier}

We now study the intrusions to the obstructed barrier. We randomly select 50 entry points, and for each entry point, we simulate 10 different intrusions at the intruding speed of 1 unit/sec. Among the 10 intrusions, one is along the optimal intruding path and others are along randomly-selected intruding paths. For each entry point, the intrusion coverage degree results are normalized over that obtained with the optimal intruding path. Fig. 9 shows the results of six selected entry points. Each point for "random intruding path" in the figure is averaged over those 9 random intrusion paths. For reader's interest, the optimal intruding path starting from entry point \#2 is plotted in Fig. 6, and in the zoomed 


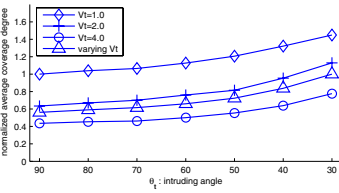

Figure 8. Intrusion coverage degree vs. intruding angle in non-obstructed barrier.

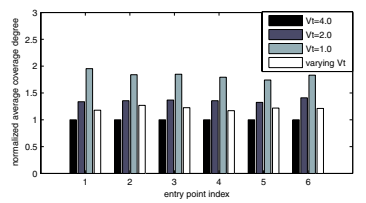

Figure 10. Comparison of intrusion coverage degree in the obstructed barrier (various intruding speeds).

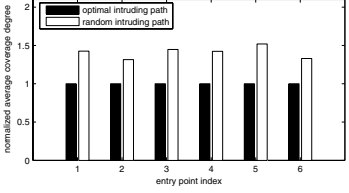

Figure 9. Comparison of intrusion coverage degree with different intruding paths.

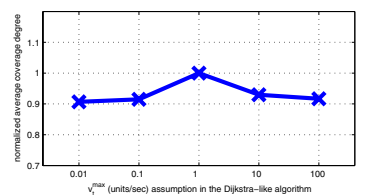

Figure 11. Intrusion coverage degrees for various sensor movement strategies $\left(v_{t}=1\right.$ units/sec).

portion of the figure, the movement strategy of each sensor is shown as two-directional line segments. Clearly, a smart intruder shall follow the optimal intruding path which always yields lower intrusion coverage degree than a random path.

We now examine the effect of intruding speed. For each of the 50 entry points, we also simulate intrusions along the optimal intruding path but at various speeds. The results are normalized over that obtained with intruding speed of 4 units/sec. Fig. 10 shows that a smart intruder shall always move at the maximum intruding speed, in order to minimize the chance of being detected, which is consistent with our earlier observation in Fig. 8.

Recall that $\bar{f}_{\Theta_{s}}^{\max }$ was obtained by assuming the knowledge of maximum intruding speed. However, if such knowledge is not available to the sensor network and sensors move according to, unfortunately, a false-optimal movement strategy derived with a wrong assumption on the maximum intruding speed, the coverage performance will be inevitably worse that of $\bar{f}_{\Theta_{s}}^{\max }$. This is because, with regard to the true maximum intruding speed, such sensor movement is merely another non-optimal strategy. This phenomenon can be clearly observed from Fig. 11 and confirms our analysis in Section 4.2. In general, more knowledge about the intrusion characteristics helps in designing sensor movement strategies to better defend an obstructed barrier.

\section{Conclusions}

In this paper, we investigate the coverage performance of a mobile sensor network in defending against barrier intrusions. More specifically, we define the coverage degree of an intrusion as the number of distinct sensors that each detects the intruder before it reaches the destination side of the barrier, and then study the best worst-case performance of a mobile sensor network in terms of the intrusion coverage degree at both non-obstructed and obstructed barriers.

For non-obstructed barriers, we prove that the optimal sensor movement strategy is that each sensor moves in parallel to the destination side of the barrier, and the optimal intrusion is

to intrude the barrier at the maximum speed along the path that is perpendicular to the destination side of the barrier. For obstructed barriers, we identify the unique obstacle eclipse effect that can only be observed in obstructed barriers, and then propose a Dijkstra-like algorithm to generate a sensor movement strategy that performs well in defending obstructed barriers. In-depth simulation verifies the correctness of our theoretical analysis and demonstrates the effectiveness of our devised sensor movement strategies.

\section{References}

[1] Qualnet simulator. http://www.scalable-networks.com/. Online Link.

[2] M. Batalin and G. Sukhatme. Spreading out: A local approach to multi-robot coverage. 6th International Conference on Distributed Autonomous Robotic Systems (DSRSO2), 2002.

[3] N. Bisnik, A. Abouzeid, and V. Isler. Stochastic event capture using mobile sensors subject to a quality metric. Proceedings of ACM MobiCom, pages 98-109, 2006.

[4] M. S. Braasch and A. V. Dierendonck. GPS Receiver Architectures And Measurements. Proceedings of the IEEE, 87(1):48-64, Jan. 1999.

[5] Q. Cao, T. Yan, J. Stankovic, and T. Abdelzaher. Analysis of Target Detection Performance for Wireless Sensor Networks. IEEE ICDCS, June 2005.

[6] T. Clouqueur, V. Phipatanasuphorn, P. Ramanathan, and K. K. Saluja. Sensor Deployment Stategy for Target Detection. 1st ACM International Workshop on Wireless Sensor Networks and Applications, 2002.

[7] O. Dousse, C. Tavoularis, and P. Thiran. Delay of Intrusion Detection in Wireless Sensor Networks. Proceedings of ACM MobiHoc, pages 155-165, May 2006.

[8] S. C. Fisher and K. Ghassemi. GPS IIF-The Next Generation. Proceedings of the IEEE, 87(1):24-47, Jan. 1999.

[9] R. Gau and Y. Peng. A dual approach for the worst-case-coverage deployment problem in ad-hoc wireless sensor netowrks. Proceedings of IEEE MASS, pages 427-436, Oct. 2006.

[10] C. Gui and P. Mohapatra. Power Conservation and Quality of Surveillance in Target Tracking Sensor Networks. Proceedings of ACM MobiCom, Sept. 2004.

[11] P. Hall. Introduction to the Theory of Coverage Process. John Wiley \& Sons, 1988.

[12] A. Howard, M. Mataric, and G. Sukhatme. Mobile Sensor Network Deployment Using Potential Fields: A Distributed, Scalable Solution to the Area Coverage Problem. 6th International Conference on Distributed Autonomous Robotic Systems (DSRSO2), 2002.

[13] L. Hu and D. Evans. Localization for Mobile Sensor Networks. Proceedings of ACM MobiCom, pages 45-57, 2004.

[14] J. C. Jr and G. L. Stuber. Nonlinear Multiuser Parameter Estimation and Tracking in CDMA Systems. IEEE Transactions on Communications, 48(12):2053-2063, Dec 2000

[15] S. Kumar, T. H. Lai, and A. Arora. Barrier Coverage With Wireless Sensors. Proceedings of ACM MobiCom, pages 284-298, 2005.

[16] S. Kumar, T. H. Lai, and J. Balogh. On k-Coverage in a Mostly Sleeping Sensor Network. Proceedings of MobiCom, pages 144-158, 2004.

[17] B. Liu, P. Brass, O. Dousse, P. Nain, and D. Towsley. Mobility Improves Coverage of Sensor Networks. Proceedings of ACM MobiHoc, pages 300-308, 2005.

[18] B. Liu and D. Towsley. A Study of the Coverage of Large-scale Sensor Networks. IEEE International Conference on Mobile Ad-hoc and Sensor Systems (MASS), pages $475-483$, Oct. 2004

[19] S. Meguerdichian, F. Koushanfar, M. Potkonjak, and M. B. Srivastava. Coverage Problems in Wireless Ad-hoc Sensor Networks. IEEE Infocom, pages 1380-1387, 2001.

[20] S. Meguerdichian, F. Koushanfar, G. Qu, and M. Potkonjak. Exposure In Wireless Ad-Hoc Sensor Networks. Proceedings of ACM MobiCom, pages 139-150, July 2001.

[21] N. Patwari and A. O. H. III. Using Proximity and Quantized RSS for Sensor Localization in Wireless Networks. IEEE/ACM 2nd Workshop on Wireless Sensor Nets. \& Applications, 2003

[22] J. Pearce, P. Rybski, S. Stoeter, and N. Papanikolopoulous. Dispersion Behaviors for a Team of Multiple Miniature Robots. IEEE ICRA, 2003.

[23] N. B. Priyantha, A. Chakraborty, and H. Balakrishnan. The Cricket Location-Support System. Proceedings of ACM MobiCom, pages 32-43, Aug. 2000

[24] S. Shakkottai, R. Srikant, and N. Shroff. Unreliable Sensor Grids: Coverage, Connectivity and Diameter. Proceedings of IEEE Infocom, 2:1073-1083, 2003.

[25] M. Shaw, P. Levin, and J. Martel. The Dod: Stewards Of A Global Information Resource, The Navstar Global Positioning System. Proceedings of the IEEE, 87(1):16-23, Jan. 1999.

[26] X. Wang, G. Xing, Y. Zhang, C. Lu, R. Pless, and C. Gill. Integrated coverage and connectivity configuration in wireless sensor networks. SenSys, pages 28-39, November 2003

[27] G. Yang, W. Zhou, and D. Qiao. Defending the Barrier with Mobile Sensors: A Game Theoretic Approach. Technical report, Iowa State University, 2006.

[28] H. Zhang and J. C. Hou. Is Deterministic Deployment Worse than Random Deployment for Wireless Sensor Networks? Proceedings of IEEE Infocom, March 2006. 